Spatial and temporal analysis of laser induced plasma from a polymer sample

This article has been downloaded from IOPscience. Please scroll down to see the full text article.

1993 J. Phys. D: Appl. Phys. 2635

(http://iopscience.iop.org/0022-3727/26/1/007)

View the table of contents for this issue, or go to the journal homepage for more

Download details:

IP Address: 117.211.83.202

The article was downloaded on 29/10/2011 at 07:26

Please note that terms and conditions apply. 


\title{
Spatial and temporal analysis of laser induced plasma from a polymer sample
}

\section{G Padmaja, A V Ravi Kumar, P Radhakrishnan, V P N Nampoori and C P G Vallabhan}

Laser Division, Department of Physics, Cochin University of Science and

Technology, Cochin 682 022, Kerala, India

Received 5 March 1992, in final form 4 August 1992

\begin{abstract}
Spatial and temporal analyses of the spectra of the laser induced plasma from a polytetrafluroethylene (PTFE) target obtained with the $1.06 \mu \mathrm{m}$ radiation from a Q-switched Nd:YAG laser have been carried out. The spatially resolved spectra of the plasma emission show that molecular bands of $C_{2}$ (Swan bands) and $\mathrm{CN}$ are very intense in the outer regions of the plasma, whereas higher ionized states of carbon are predominant in the core region of the plasma emission. The vibrational temperature and population distribution in the different vibrational levels have been studied as a function of laser energy. From the time resolved studies, it has been observed that there exist fairly large time delays for the onset of emission from all the species in the outer region of the plasma. Also, the molecular bands in each region exhibit much larger time delays in comparison to the ionic lines in the plasma.
\end{abstract}

\section{Introduction}

The interaction of high power laser pulses with organic materials has been investigated extensively over the past two decades. It has been shown by several groups of workers that the irradiation of polymer surfaces with laser pulses can lead to the precise removal of material in a geometry that is defined by the light beam [1]. Most of these investigations have clearly demonstrated the potential use of this process in technology and medicine. The ablation of polymeric materials and biological tissues by UV laser irradiation has direct applications in microfabrications and in microsurgery respectively as it provides a means for precise removal of material with high spatial resolution [2]. There has also been recent interest in the use of polymers for optical materials and in the laser machining of polymers via ablative photodecomposition [3].

When pulsed IR laser radiation falls on the surface of an organic substance, the material on the surface layer is spontaneously etched away to a depth which can be controlled by the number of pulses and fluence of the beam. Such controlled etching has important applications in photolithography [4]. It has been suggested that 'ablative photodecomposition' occurs as a result of direct bond scission by the multiphoton processes induced by laser photons $[5,6]$. The resultant energetic molecular fragments then rapidly ablate from the irradiation sites [7]. It is shown that laser pulses from IR lasers can produce fast heating accompanied by surface damage [8]. Under such excitation, the photochemistry is simplified and the ablation process is best described as explosive thermal decomposition [9]. The use of IR lasers gives rise to multiphoton excitations over the vibrational levels of the ground electronic states which are then followed by thermal decomposition.

The $\mathbb{R}$ radiation from a high power laser pulse usually generates intense plasma emission from the target surface. Different kinds of studies of laser induced plasma, such as specular reflection of the laser light, second-harmonic generation, charge and velocity distribution of ablated species, ablation pressure, $\mathrm{x}$-ray emission, etc., using moderately high power IR laser pulses having peak powers $\sim 10^{14} \mathrm{~W} \mathrm{~cm}^{-2}$ have been investigated in detail by many investigators [10-13]. The nature and characteristics of the laser induced plasma (LIP) from a solid target depend on various parameters like the chemical composition of the target [14], wavelength of radiation [15], energy deposited on the target, pressure of the residual gas in the plasma chamber, etc. The composition of the plasma will in general depend on its distance from the sample surface as a result of the rapid expansion of the plasma from the target fallowed by the lowering of the plasma temperature. The populations of the various types of molecular, ionic and neutral species in the plasma will also depend on the spatial separation of the point of 


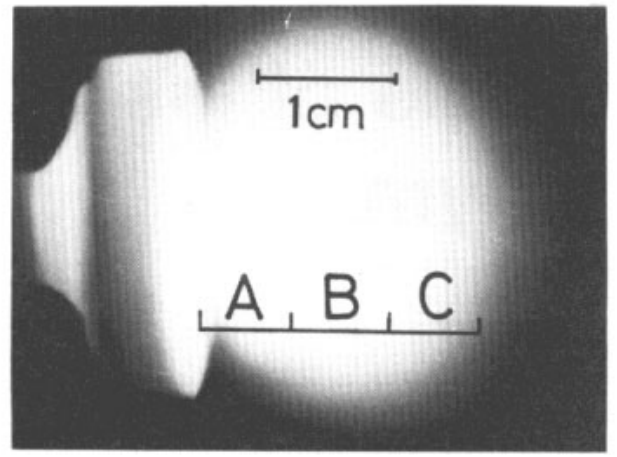

Figure 1. Photograph of the laser induced plasma emission from PTFE at a laser energy density of $\sim 3 \mathrm{~J} \mathrm{~cm}^{-2}$ showing the regions of the plasma studied.

observation from the target. Based on the physical composition LIP emission can thus be divided into three broad spatial regions, namely:

(1) the central or the core region of the plasma emission close to the sample surface, where the temperature is maximum and most of the species consist of ionized atoms;

(2) the mid region, where apart from ionized species, both neutral and a certain number of molecular species are present;

(3) the extended region, in which the plasma temperature is comparatively lower and larger number densities of molecular species occur.

Optical spectroscopy is an effective as well as convenient tool to detect various transient species such as excited atoms, ions and diatomic/polyatomic molecules (which form the third category of products), all of which are produced during laser ablation. It has been pointed out that the excited diatomic species, which are the sources of band emission in the different regions of the plasma, may be formed by a secondary photolysis of the initial products of the laser ablation process. Laser induced plasma spectroscopy (LIPS) has been successfully used in the present study to probe transient species such as $\mathrm{C}_{2}$ and $\mathrm{CN}$ in the laser ablation process of polymers. We report here the results of the spectroscopic as well as the time resolved analysis of laser induced plasma emission from PTFE (polytetrafluroethylene). In the photoabiation of polymers, knowledge of the time evolution of the ablation process is of considerable significance since it may, in conjunction with theoretical models [16], help to resolve uncertainties in the interaction mechanism and thus lead to improved understanding of this phenomenon which has important practical applications [17]. The time delays and decay times of the emission from different species in the three distinct regions of the plasma have been measured in the present study. The results throw much light on the complex recombination process at work in a laser induced plasma.
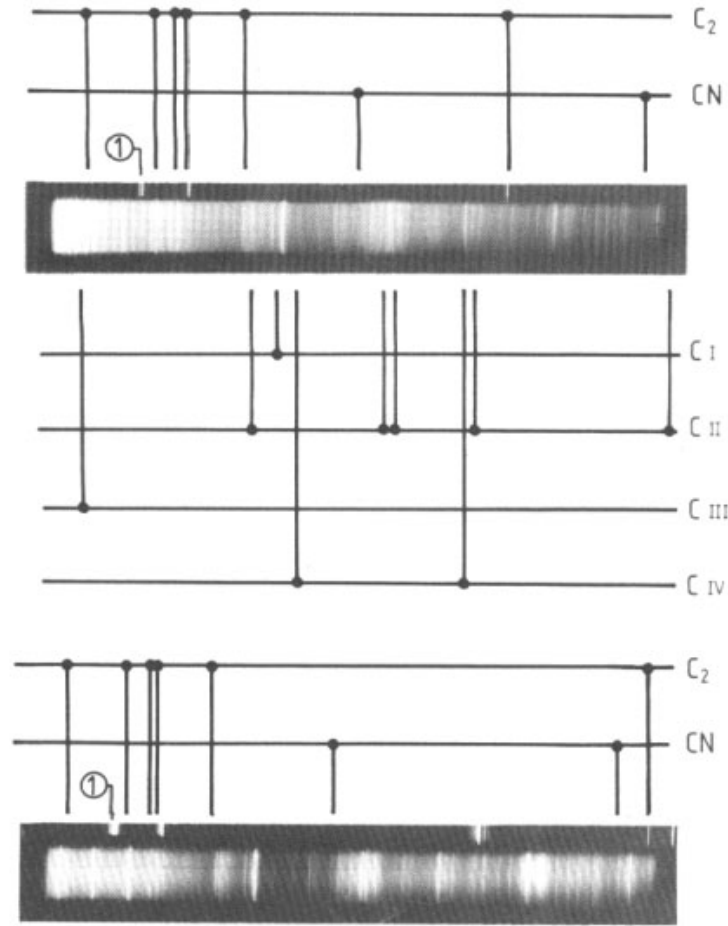

(b)

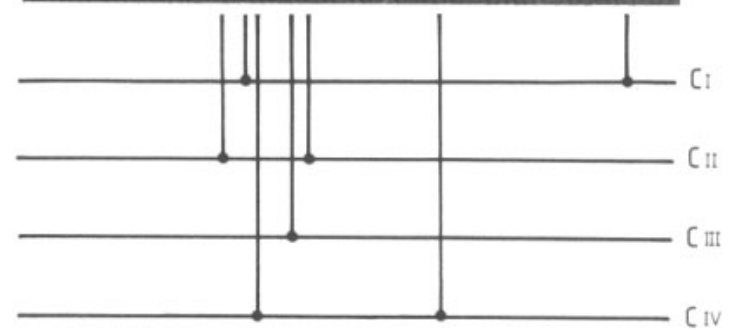

$C_{2}$ Swan bands

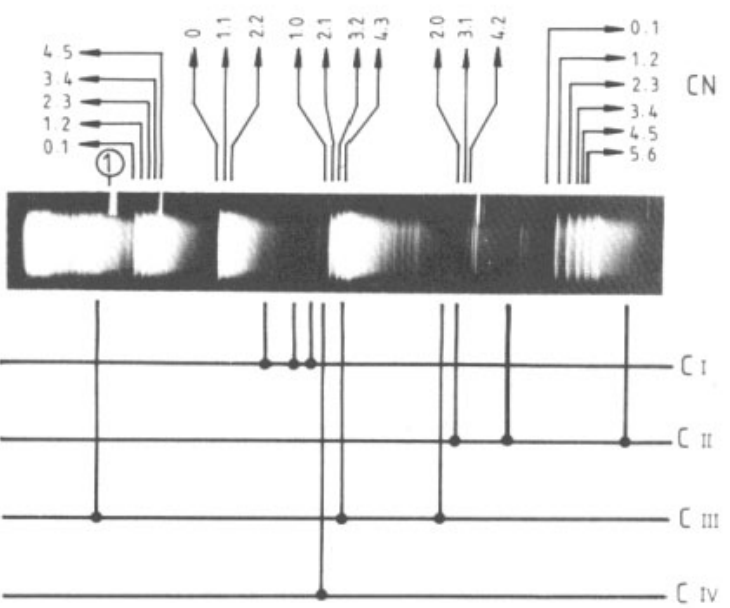

Figure 2. The emission spectrum from the different regions of the laser induced plasma from PTFE: (a) central region A, (b) mid-region $B,(c)$ extended region C. 1, Mercury reference $\lambda=579.0 \mathrm{~nm}$. Laser energy density $\sim 4 \mathrm{~J} \mathrm{~cm}^{-2}$.

\section{Experimental technique}

Plasma was generated (figure 1) by laser ablation of the sample (PTFE) using a $1.06 \mu \mathrm{m}$ laser beam from a Q-switched Nd: YAG laser (Quanta Ray DCR-11) 


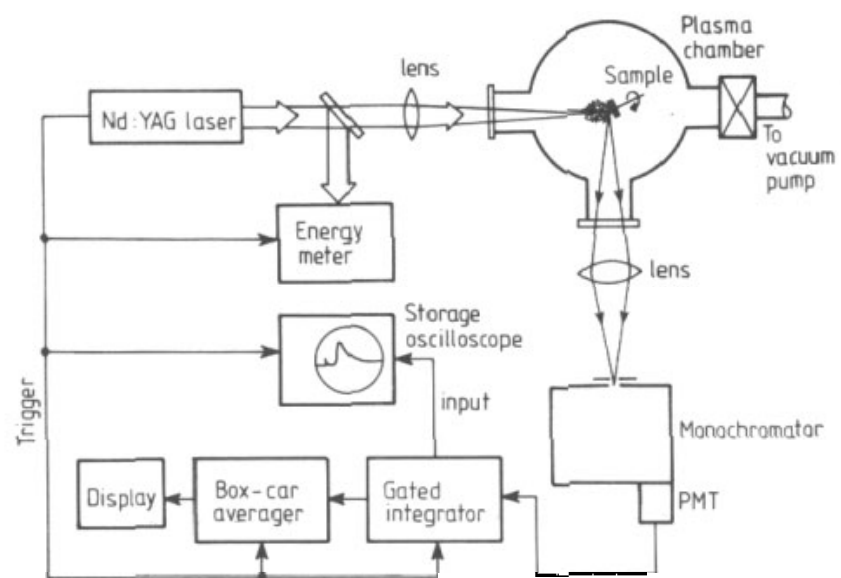

Figure 3. Schematic diagram of the experimental set-up for time resolved analysis of the plasma emission.
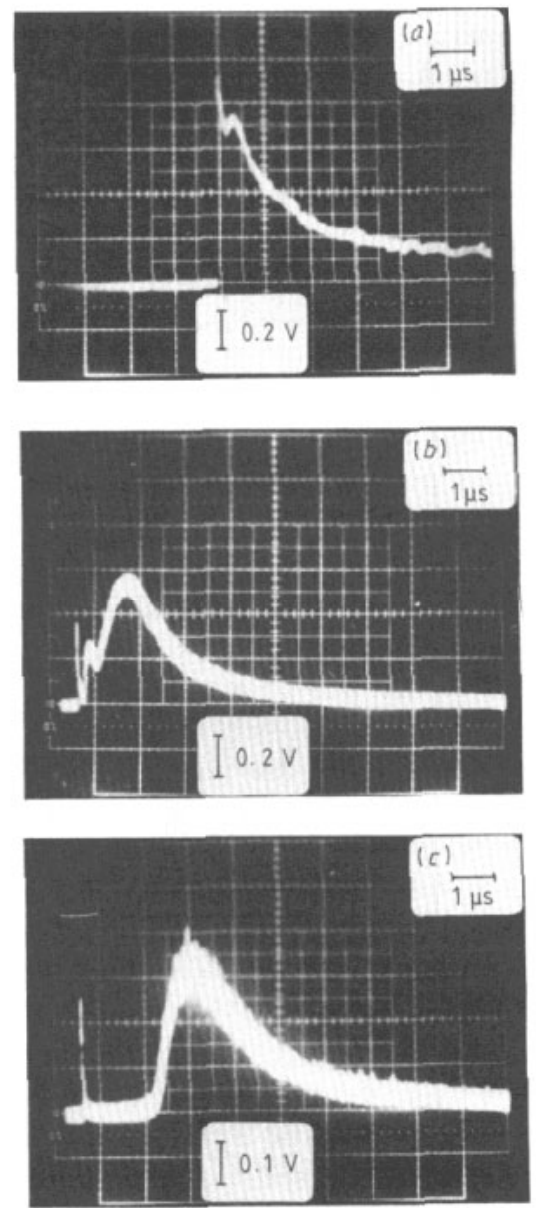

Figure 4. Typical oscilloscope trace of the PMT response to the plasma emission from the $C_{2}$ species (at $\lambda=562.1 \mathrm{~nm}$ ) from the different regions of the plasma: (a) central region $A,(b)$ mid-region $B$, (c) extended region $C$.

having a pulse width of $\sim 10 \mathrm{~ns}$ at $10 \mathrm{~Hz}$ repetition rate. The sample in the form of a disc $(2.5 \mathrm{~cm}$ diameter and $5 \mathrm{~mm}$ thickness) was placed in a partially evacuated (pressure $\sim 200 \mathrm{~m}$ Torr) plasma chamber provided with quartz windows. The laser beam was focused to a dia- meter of $\sim 1 \mathrm{~mm}$ onto the target, which is rotated about the axis of the target disc to avoid non-uniform pitting of the target surface. The bright plasma emission was observed through a side window at $90^{\circ}$ with respect to the laser beam axis. The spectra from the three distinct regions, namely, the central (A), middle (B) and extended (C) regions of the plasma were recorded photographically using a Carl-Zeiss three prism spectrograph and appropriate optics for focusing [18]. The plasma emission spectra of PTFE from the three different regions are shown in figures $2(a),(b)$ and $(c)$. The wavelengths of the emissions of the different atomic and molecular species in the plasma were determined from the recorded spectra using standard spectral data sources $[19,20]$.

In the time resolved analysis, a part of the plasma plume was focused onto the slit of a monochromator (McPherson, $0.2 \mathrm{~m}$ ) coupled to a photomultiplier tube (PMT) (Hamamatsu R446, 3 ns rise time) and a storage oscilloscope/box-car averager. In order to study the time evolution of a particular species produced by laser ablation, the characteristic lines were selected using the monochromator, and the PMT output terminated at $50 \Omega$ resistance, was fed to a $100 \mathrm{MHz}$ storage oscilloscope (Tektronix Model 466) (figure 3) [21]. Typical oscilloscope (CRO) traces of the PMT response to the emission from the $C_{2}$ species in the three different regions ( $A, B$ and $C$ ) of the plasma plume are shown in figures $4(a),(b)$ and $(c)$ respectively. The amplitudes of the pulse shapes were monitored by a gated integrator and box-car averager (Stanford Research Systems Model SR250).

\section{Results and discussions}

As can be seen from the spectra, (figures $2(a),(b)$ and (c)) corresponding to the three regions, the recorded results show distinctly different characteristics. The emission lines due to higher ionization states of carbon (C I, C II, C III and C IV) are clearly seen in the core and mid regions of the plasma (figures $2(a)$ and $2(b)$ ). Molecular bands corresponding to the $A^{3} \Pi_{g}-X^{3} \Pi_{u}$ transitions of $\mathrm{C}_{2}$ (Swan bands) [19] in the wavelength range from 500 to $590 \mathrm{~nm}$ are predominant in the spectrum of the extended region. The reaction of the excited carbon with the nitrogen content of the air remaining in the chamber generates $\mathrm{CN}$ molecules in the plasma. The presence of the characteristic $\mathrm{B}^{2} \Sigma^{+}-\mathrm{X}^{2} \Sigma^{+}$bands of the $\mathrm{CN}$ molecule in the violet region of the spectrum is a clear indication of the occurrence of such a process.

The relative intensities of the vibrational bands were obtained using a microdensitomer (Carl-Zeiss). The intensity distribution in a band can be used to determine the vibrational temperature of the plasma emission source. According to the vibrational sum rule for the intensities of different bands in a progression, the sums of the band strengths of all bands with the 


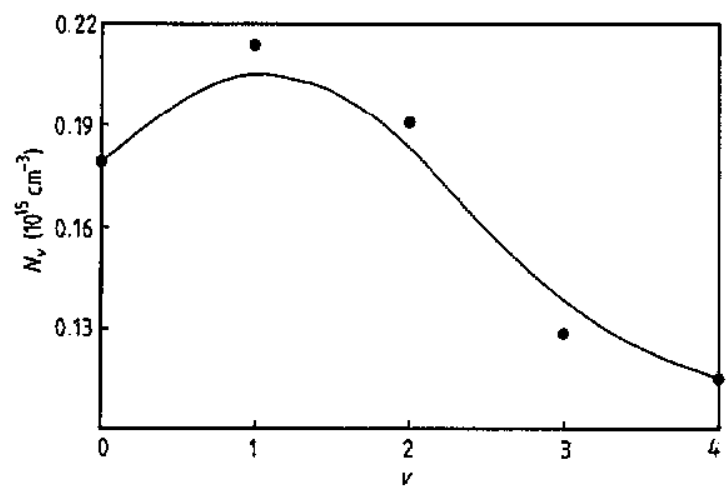

Figure 5. The vibrational distribution in the $\mathrm{C}_{2}$ Swan bands $(562.1 \mathrm{~nm})$ in region $C$ of the plasma. (Laser energy of $\sim 68 \mathrm{~mJ}$.)

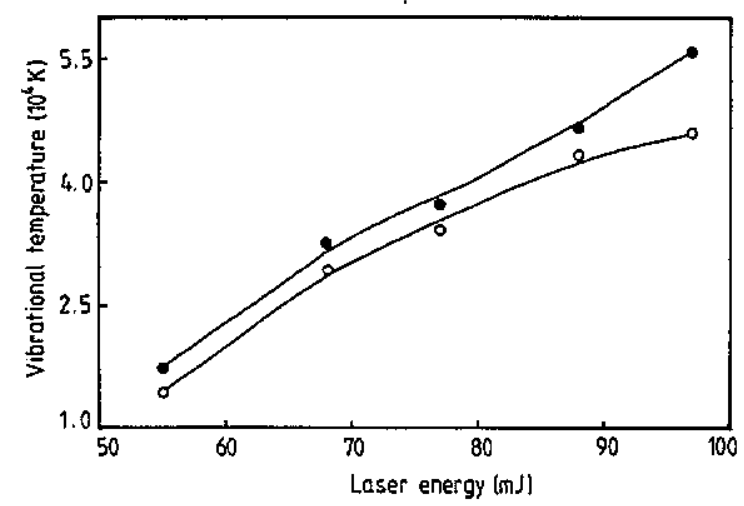

Figure 6. The variation of the vibrational temperature of the $\mathrm{C}_{2}$ Swan bands $(O)$ and $\mathrm{CN}$ bands $(\mathrm{O})$ in region $\mathrm{B}$ of the plasma emission with laser energy.

same upper or lower state are proportional to the number of molecules in the respective states [22], i.e.

$$
\begin{aligned}
& \sum_{v^{*}} \frac{I\left(v^{\prime}, v^{\prime \prime}\right)}{\nu^{4}} \propto N_{v^{\prime}} \\
& \sum_{v^{\prime}} \frac{I\left(v^{\prime}, v^{\prime \prime}\right)}{\nu^{4}} \propto N_{v^{*}}
\end{aligned}
$$

where $I\left(v^{\prime}, v^{\prime \prime}\right)$ is the emission intensity from the vibrational level $v^{\prime}$ of the upper electronic state to the vibrational level $v^{\prime \prime}$ of the lower electronic state, $\nu$ is the frequency in $\mathrm{cm}^{-1}, N_{v^{\prime}}$ and $N_{v^{\prime \prime}}$ the numbers of molecules in the vibrational level of the upper and lower electronic state respectively.

In thermal equilibrium, the number densities at various vibrational levels of a molecule in the excited state can be evaluated using the formula

$$
\log \sum_{v^{\prime \prime}} \frac{I\left(v^{\prime}, v^{\prime \prime}\right)}{v^{4}}=C-\frac{G^{\prime}\left(v^{\prime}\right) h c}{k_{\mathrm{B}} T}
$$

where $h$ is Planck's constant, $c$ the velocity of light, $k_{\mathrm{B}}$ Boltzmann's constant, $C$ a constant, $G^{\prime}\left(v^{\prime}\right)$ the term value corresponding to the excited state vibrational levels and $T$ is the vibrational temperature. Therefore by plotting the logarithms of the sums of the band strengths measured in the various $v^{\prime \prime}$ or $v^{\prime}$ progressions against the vibrational term values $G(v)$ a straight line is obtained. While the intercept $C$ is of no significance, the slope is a direct measure of the vibrational temperature [23]. The vibrational distributions in the

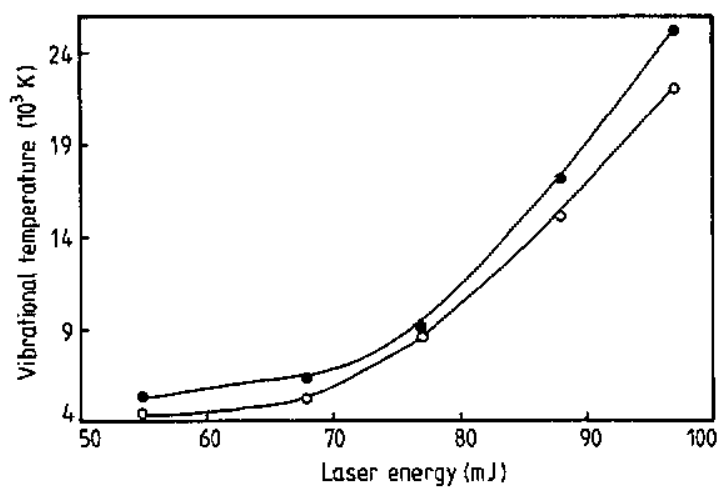

Figure 7. The variation of the vibrational temperature of the $\mathrm{C}_{2}$ Swan bands $(O)$ and $\mathrm{CN}$ bands $(\mathrm{O})$ in region $\mathrm{C}$ of the plasma emission with laser energy.

excited states of the $C_{2}$ molecule are shown in figure 5. Similar distributions were observed in the case of $\mathrm{CN}$ bands also. The inverse distribution observed for $v<2$ in the case of the $\mathrm{C}_{2}$ molecule is in accordance with the Franck-Condon principle. Similar distributions have been observed in certain other molecules also $[24,25]$.

The variations of vibrational temperature with laser energy corresponding to the $\mathrm{C}_{2}$ Swan bands and $\mathrm{CN}$ molecules in the mid-region (B) of the plasma emission are shown in figure 6 . The vibrational temperature was found to vary from $1.75 \times 10^{4} \mathrm{~K}$ to $5.6 \times 10^{4} \mathrm{~K}$ for $\mathrm{C}_{2}$ molecules and from $1.45 \times 10^{4} \mathrm{~K}$ to $4.6 \times 10^{4} \mathrm{~K}$ for $\mathrm{CN}$ molecules in the mid-region as the laser energy is varied from $55 \mathrm{~mJ}$ to $98 \mathrm{~mJ}$. This variation of vibrational temperature with laser energy is essentially due to the fact that, as the incident beam power increases, a comparatively larger number of molecules are excited into the higher vibrational states. The knee appearing in figure 7 is evidently due to a threshold-like phenomenon resulting from the possible nonlinear interactions such as self-focusing of the laser beam within the plasma medium. The Debye length, which is the characteristic screening length of the plasma, is given by $[26,27,31]$

$$
\lambda_{\mathrm{D}}=\left(\frac{k_{\mathrm{B}} T_{0}}{8 \pi N_{0} e^{2}}\right)^{1 / 2}
$$

where $k_{\mathrm{B}}$ is the Boltzmann constant, $T_{0}$ the equilibrium plasma temperature $\left(\sim 10^{5} \mathrm{~K}\right), N$ the equilibrium concentration $\left(\sim 10^{12} \mathrm{~cm}^{-3}\right)$ and $e$ the electron charge.

It is known that the self-focusing phenomenon of laser beams in plasma will be predominant if the Debye length is less than the beam diameter. In our case, $\lambda_{\mathrm{D}} \approx 3 \mu \mathrm{m}$ which is much less than the beam diameter $(\sim 1 \mathrm{~mm})$. Such self-focusing of the laser beam in the plasma leads to an enhanced effective power density resulting in more intense emission. The threshold for this nonlinear effect for region $C$ is clearly seen in figure 7 and it occurs at $\sim 70 \mathrm{~mJ}$. It is interesting to note that such a threshold-like phenomenon is not explicitly evident in the case of region B (figure 6). Apparently, this is due to the fact that a greater laser energy density due to self-focusing and a larger plasma density and 
temperature lead to a much lower value for this threshold in region $B$ which is near the saturation region. Similarly, the variation of vibrational temperature for the $\mathrm{C}_{2}$ Swan bands and $\mathrm{CN}$ molecules in the extended region $(C)$ of the plasma emission with laser energy is shown in figure 7 . Here, the vibrational temperature was found to vary from $5.33 \times 10^{3} \mathrm{~K}$ to $2.25 \times 10^{4} \mathrm{~K}$ for $\mathrm{C}_{2}$ molecules and from $5.23 \times 10^{3} \mathrm{~K}$ to $2.32 \times 10^{4} \mathrm{~K}$ for $\mathrm{CN}$ molecules in the extended region as the laser energy is varied from $55 \mathrm{~mJ}$ to $98 \mathrm{~mJ}$. In this region, which is comparatively cooler, the recombination processes dominate, and this could also contribute to an increase in the vibrational temperature of the molecules as the laser energy is increased.

Figures $4(a),(b)$ and $(c)$ show the oscilloscope traces of the PMT response of the emissions from the $\mathrm{A}, \mathrm{B}$ and $\mathrm{C}$ regions of the plasma emission. The CRO trace shows a sharp, 'prompt' emission and a delayed emission peaking after a few microseconds $[28,29]$. The 'prompt' emission could easily result from the ablated species ejected from the target surface. On the other hand, the delayed emission is most likely to be due to electronic and/or molecular collisional excitations [28]. From the measured value of the time delay exhibited by the PMT signal trace of the emission (figure $4(\mathrm{c})$ ), the velocity of the species is obtained and the corresponding energy calculated is found to be $\sim 6 \mathrm{eV}$. Since the energy of the ablated species is much greater than $1 \mathrm{eV}$, in all probability the delayed emission is due predominantly to the collisional excitation rather than to the evaporated species. From the above figures, it is obvious that there is a notable time delay between the incidence of the laser pulse (all time delay measurements are taken with respect to the 'prompt' emission) on the target and the onset of emission from a particular species in the three different regions of the plasma. This is due to the fact that in the core of the plasma (A), the plasma temperature is a maximum and most of the species consist of ionized atoms which results in the smaller time delay (of the order of nanoseconds) of emission. In region B of the plasma, apart from the ionized species, both neutral and a certain number of molecular species are present due to recombination and other allied processes, which results in a comparatively larger time delay of emission (of the order of microseconds) as compared to that of the core region of the plasma. The emission is still further delayed in region $\mathrm{C}$ of the plasma where molecular bands predominate. It must be noted that the overall optical emission begins only about $0.5 \mu$ s after the start of the laser pulse. Figures $8(a),(b)$ and $(c)$ show the variation of the radiation emission time delays of certain identified species in the three different plasma regions with laser energy.

From the time delays observed, it is seen that in all three different regions of the plasma, the molecular band $\left(\mathrm{C}_{2}\right.$ and $\left.\mathrm{CN}\right)$ emissions have much larger time delays as compared to the emission from ionic/neutral species. From such time delays it is also observed that

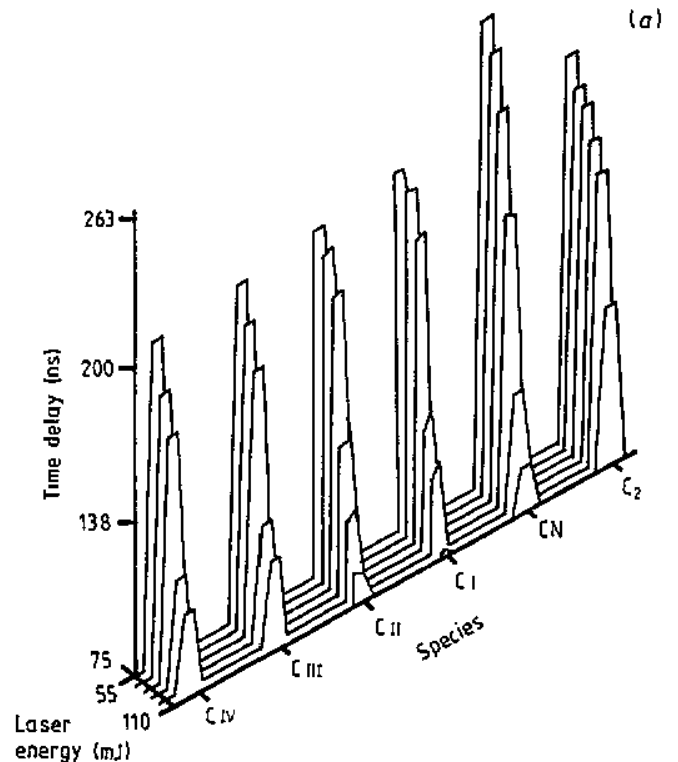

(a)

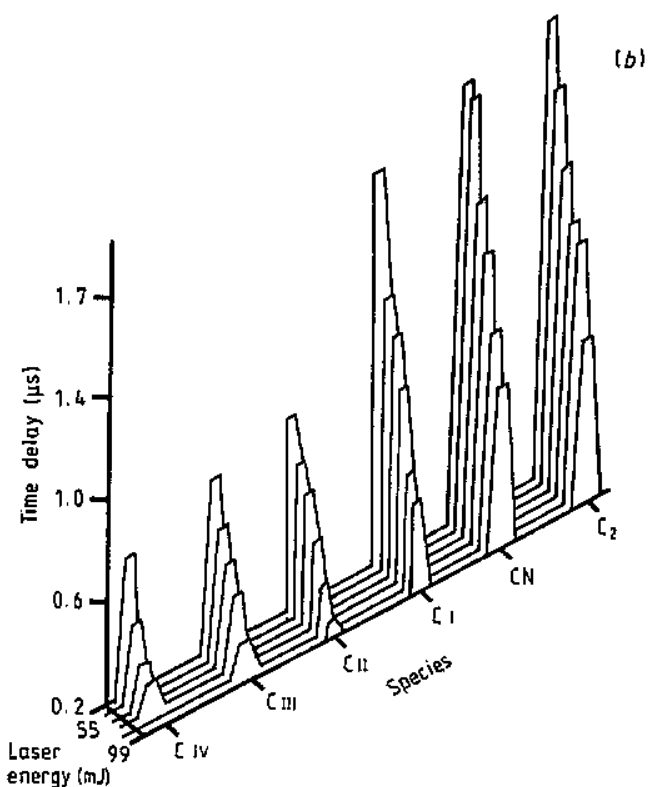

(b)

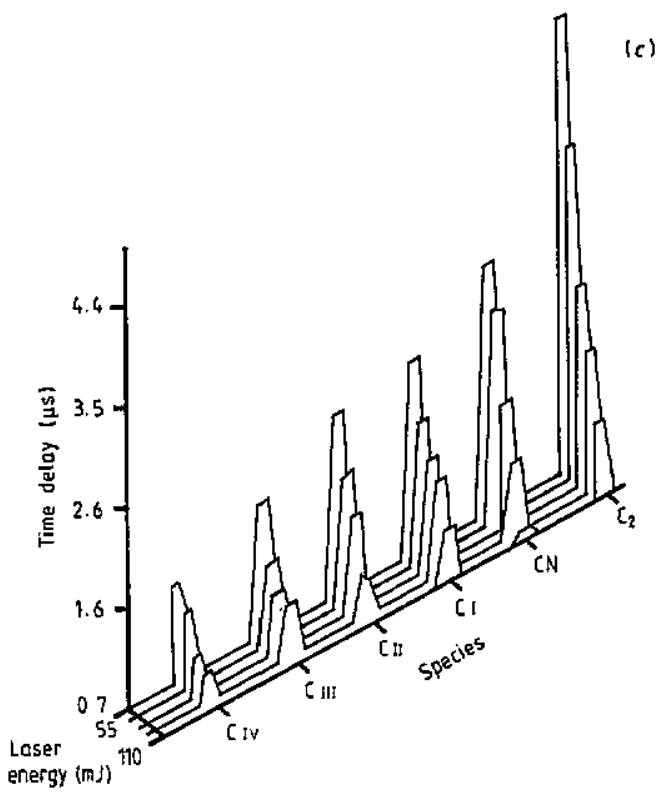

Figure 8. Variation of the time delays of emission of the different species with laser energy for regions $\mathrm{A}(a), \mathrm{B}(b)$ and $\mathrm{C}(\mathrm{c})$ of the plasma. 


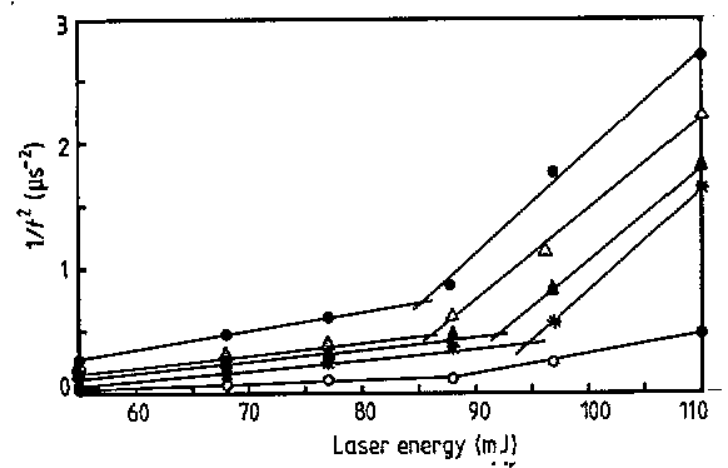

Figure 9. Variation of $1 / t^{2}$ with laser energy of different species in region $C$ of the plasma: $C$ IIt $(O), C \|(\Delta), C$ । (A), CN (*), $\mathrm{C}_{2}(\mathrm{O})$.

the spectral lines of higher ionized species are first to appear and these are followed in turn by those of successively lower states of ionization [30]. In general, there is a decrease in time delay as the laser energy increases. There are many reasons for the occurrence of a time delay for emission from any species. Prominent among them are: (1) time-of-flight of the species, (2) thermal processes leading to generation of plasma from the target, (3) recombination and/or dissociation of the species. As a consequence of the nonlinear effect mentioned earlier, the enhancement of the effective power density of the laser beam in the plasma will cause a corresponding increase in the velocity of the species, thereby decreasing the delay of emission. This can result in the sudden change of slope at the threshold as seen in figure 9. Variation of the time-offlight of the different species from the laser induced plasma (LIP) of a Plexiglass target using $30 \mathrm{ps}, 1.06 \mu \mathrm{m}$ wavelength Nd:YAG laser pulses was reported by Rohr et al [11]. If the time-of-flight alone were responsible, we would expect the time delay to be proportional to $M^{1 / 2}$ ( $M$ is the mass of the species) which is not found to be the case with the present results. For example, the various ions of carbon have different time delays, which should have been the same if the time delay were proportional to $M^{1 / 2}$. If we assume that the plasma temperature is proportional to the laser energy $E$, then one would expect $E$ to be proportional to $1 / t^{2}$, where $t$ is the time delay of emission for a particular species. This is not in accordance with the observations as seen in figure 9 , which shows the variation of $1 / t^{2}$ with laser energy for different species in the extended region of the plasma. This also supports the conclusion that the time-of-flight is not the major cause of the emission delay. Variations of $t$ with laser energy for other species in different regions of the plasma do not differ much from what is shown in figure 9.

The variation of the decay time of the emissions from different species with laser energy in the three regions are shown in figures $10(a),(b)$ and $(c)$. It is also seen that the molecular bands have a larger decay time as compared to the ionic species. As the laser energy increases, the thermal diffusion processes remaining unchanged, the plasma generation becomes
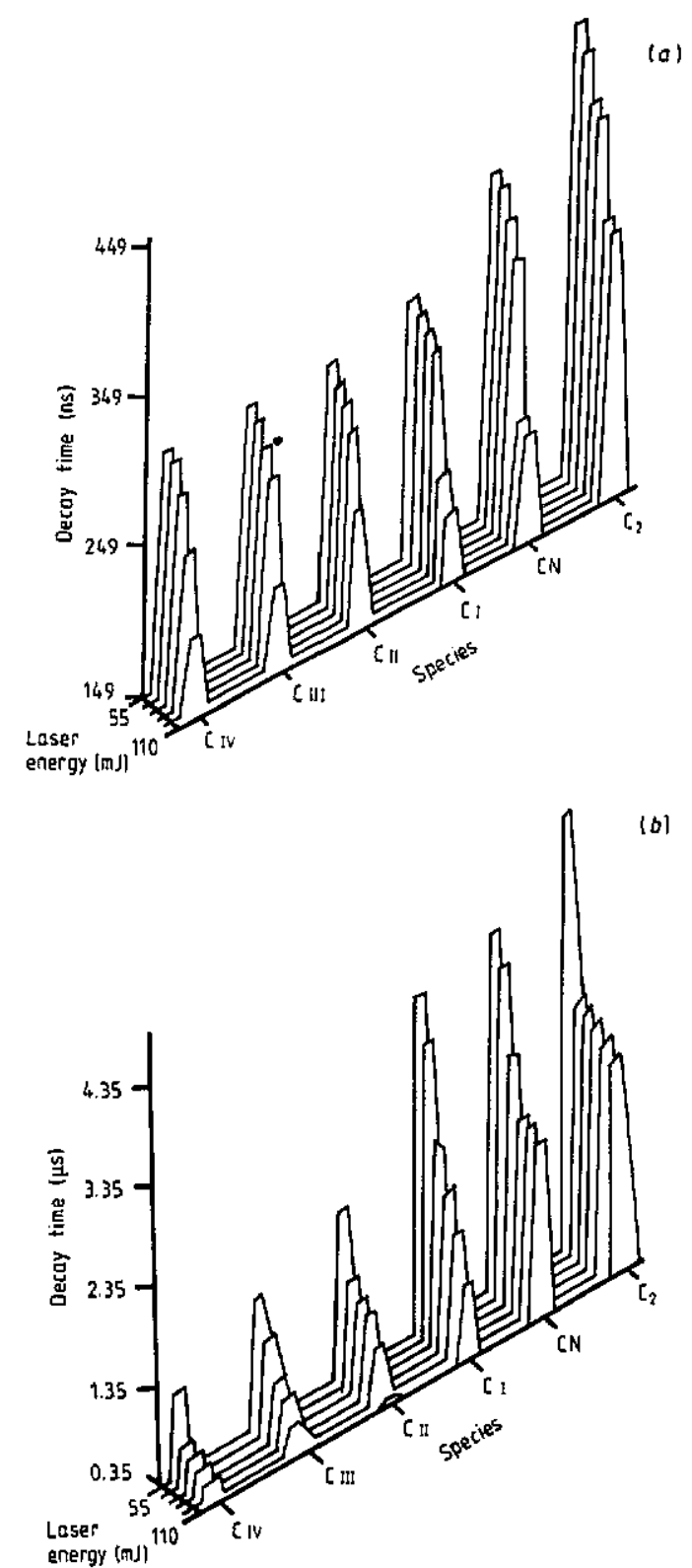

$|b|$

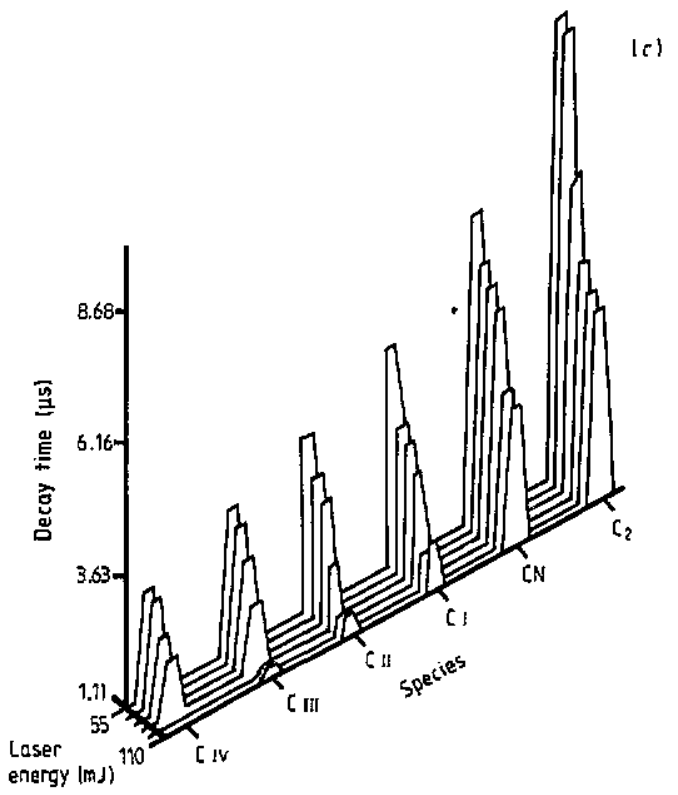

Figure 10. Variation of the decay times of emission of the different species with laser energy for regions A (a), B (b) and $\mathrm{C}(\mathrm{c})$ of the plasma. 
more rapid and we expect a decrease in the decay time of emission. Also, the increased laser energy will produce much larger densities for the various species thereby decreasing the mean free path and increasing the collision rate. All these complex processes will give rise to the variation of the time delay as well as the decay time with laser energy. An increased collision rate can also account for the decrease in decay time of emission with laser energy as observed in the present case. Such decreases in the decay time and time delay of emission with plasma temperature are found to occur as we go towards the centre or core region of the plasma.

\section{Conclusions}

The results of the spatial and temporal analysis of the laser induced plasma from a typical polymer sample of PTFE lead to several interesting conclusions related to the structure and composition of the plasma. Detailed study of the emission spectra clearly indicate the existence of molecular species like $\mathrm{C}_{2}$ and $\mathrm{CN}$ in addition to higher ionized states of carbon. From the spectroscopic studies of the emission bands, the population distribution and the vibrational temperature in different parts of the plasma plume have been obtained. The time-resolved studies of the spectral emission also throw much light on some of the complex interaction processes occurring in the plasma. From the time delays observed, in the case of higher laser energies, it can be safely concluded that the molecular bands are mainly formed when the plasma temperature begins to fall. This lends support to the view that the molecular species may not be originating from the target material at higher laser powers, but are formed as a result of recombination processes as the hot plasma cools down [21],i.e. themolecularspeciesareformedin the expanding plasma due to collisions of the atomic and ionic species expelled from the target surface. Also, the nonlinear interactions between the laser and the plasma give rise to phenomena such asself-focusing which exhibit thresholdlike characteristics.

\section{Acknowledgments}

The authors wish to thank the Department of Science and Technology and the Ministry of Human Resource Development (Government of India) for financial assistance. Two of the authors (AVRK and GP) are grateful to the Council for Scientific and Industrial Research, New Delhi, for their research fellowships.

\section{References}

[1] Srinivasan R, Braren B and Dreyfus R W $1987 \mathrm{~J}$. Appl. Phys. 61372
[2] Gorodetsky G, Kazyaka T G, Melcher R L and Srinivasan R 1985 Appl. Phys. Lett. 46828

[3] Kim H, Postlewaite J C, Zyung T and Dlott D D 1988 J. Appl. Phys. 642955

[4] Srinivasan R and Braren B 1989 Chem. Rev. 891303

[5] Srinivasan R and Mayne-Banton V 1982 Appl. Phys. Lett. 41576

[6] Srinivasan R 1983 J. Vac. Sci. Technol. B 1923

[7] Davis G M, Gower M C and Fotakis C 1985 Appl. Phys. A 3627

[8] Zyung T, Kim H, Postlewaite J C and Dlott D D 1989 Appl. Phys. Lett. 651

[9] Kim H, Postlewaite J C, Zyung T and Dlott D D 1989 Appl. Phys. Lett. 542274

[10] Dinger R, Rohr K and Weber H 1987 Laser Part. Beams 5691

[11] Rohr K, Dinger R and Weber H 1989 Laser Part. Beams 7157

[12] Balmer J E and Donaldson T P 1977 Phys. Rev. Lett. 391084

[13] Pearlmann J S, Thomas J J and Max C E 1977 Phys. Rev. Lett. 381397

[14] William R C and Brenna J T 1990 J. Chem. Phys. 92 2269

[15] Ageev V P, Akhasakhalyan A D, Gaponov S V, Gorbunov A A, Konov V I and Luchin V I 1988 Sov. Phys.-Tech. Phys. 33562

[16] Garrison B J and Srinivasan R 1985 J. Appl. Phys. 57 2909

[17] Dyer P E and Srinivasan R 1986 Appl. Phys. Lett. 48 445

[18] Padmaja G, Ravi Kumar A V, Vidyalal V, Radhakrishnan P, Nampoori V P N and Vallabhan C P G 1989 Pramana J. Phys. 32 L693

[19] Pearse R W B, Gaydon A G 1965 The Identification of Molecular Spectra (London: Chapman and Hall)

[20] William F M, Charles H Corliss and Bourdon F S 1975 Tables of Spectral Line Intensities parts I \& II (Gaithersburg, MD: National Bureau of Standards)

[21] Padmaja G, Ravi Kumar A V, Vidyalal V, Radhakrishnan P, Nampoori V P N and Vallabhan C P G 1989 J. Phys. D: Appl. Phys. 221558

[22] Herzberg G 1950 Spectra of Diatomic Molecules (Molecular Spectra and Molecular Structure 1) 2nd edn (New York: Van Nostrand)

[23] Chen X, Mazumder J and Purohit A 1991 Appl. Phys. A 52328

[24] MacDonald M A, David S J and Coombe R D $1986 J$. Chem. Phys. 845513

[25] Stephen R L, Charles W B and Alistair P R $1990 \mathrm{~J}$. Chem. Phys. 923000

[26] Sodha M S, Ghatak A K and Tripathi V K 1974 Self Focusing of Laser Beams in Dielectrics, Plasmas and Semiconductors (New Delhi: Tata McGrawHill)

[27] Shkarofsky I P, Johnston T W and Bachynski M P 1966 The Particle Kinetics of Plasmas (New York: Addison-Wesley)

[28] Wu X D, Dutta B, Hegde M S, Inam A, Venkatesan T, Chase E W, Chang C C and Howard R 1989 Appl. Phys. Lett. $\mathbf{5 4} 179$

[29] Koren G, Gupta A and Baseman R J 1989 Appl. Phys. Lett. 541920

[30] Boland B C, Irons F E and Whirter M C $1968 J$. Phys. B: At. Mol. Phys, 11180

[31] Ginzburg V L 1961 The Propagation of Electromagnetic Waves in Plasmas (Oxford: Pergamon) 